\title{
A Nonlinear Weakly Singular Retarded Henry-Gronwall Type Integral Inequality and Its Application
}

\author{
Yuanhua Lin, ${ }^{1}$ Shanhe $\mathrm{Wu},{ }^{2}$ and Wu-Sheng Wang ${ }^{1}$ \\ ${ }^{1}$ School of Mathematics and Statistics, Hechi University, Guangxi, Yizhou 546300, China \\ ${ }^{2}$ Department of Mathematics and Computer Science, Longyan University, Longyan, Fujian 364012, China
}

Correspondence should be addressed to Shanhe Wu; shanhewu@gmail.com

Received 7 March 2014; Accepted 16 August 2014; Published 26 August 2014

Academic Editor: Angelo Ciaramella

Copyright (c) 2014 Yuanhua Lin et al. This is an open access article distributed under the Creative Commons Attribution License, which permits unrestricted use, distribution, and reproduction in any medium, provided the original work is properly cited.

We establish a class of new nonlinear retarded weakly singular integral inequality. Under several practical assumptions, the inequality is solved by adopting novel analysis techniques, and explicit bounds for the unknown functions are given clearly. An application of our result to the fractional differential equations with delay is shown at the end of the paper.

\section{Introduction}

Integral inequalities play increasingly important roles in the study of existence, uniqueness, boundedness, oscillation, stability, invariant manifolds, and other qualitative properties of solutions of ordinary differential equations and integral equations. One of the best known and widely used inequalities in the study of nonlinear differential equations is GronwallBellman inequality $[1,2]$, which can be stated as follows. If $u$ and $f$ are nonnegative continuous functions on an interval $[a, b]$ satisfying $u(t) \leq c+\int_{a}^{t} f(s) u(s) d s, t \in[a, b]$, then $u(t) \leq$ $c \exp \left(\int_{a}^{t} f(s) d s\right), t \in[a, b]$. Many papers are devoted to different generalizations of Bellman-Gronwall inequality. Very well-known generalization of Bellman-Gronwall inequality to the nonlinear case is the Bihari inequality [3]. In 1956, Bihari [3] discussed the integral inequality

$$
u(t) \leq a+\int_{0}^{t} f(s) w(u(s)) d s, \quad t>0,
$$

where $a>0$ is a constant. In recent years, many researchers have devoted much effort to investigating weakly singular integral inequalities. For example, Henry [4] proposed a linear integral inequality with singular kernel to investigate some qualitative properties for a parabolic differential equation, and Sano and Kunimatsu [5] gave a modified version of Henry type inequality. However, such results are expressed by a complicated power series which are sometimes inconvenient for their applications. To avoid the shortcomings of these results, Medved' [6] presented a new method to discuss nonlinear singular integral inequalities of Henry type and their Bihari version is as follows:

$$
u(t) \leq a(t)+\int_{0}^{t}(t-s)^{\beta-1} f(s) w(u(s)) d s
$$

and the estimates of solutions are given. From then on, more attention has been paid to such inequalities with singular kernel; see [7-24] and the references cited therein. Ye and Gao [20] considered the integral inequality of Henry-Gronwall type with delay

$$
\begin{array}{r}
u(t) \leq a(t)+\int_{t_{0}}^{t}[b(s) u(s)+c(s) u(s-r)] d s, \\
t \in\left[t_{0}, T\right), \\
u(t) \leq \phi(t), \quad t \in\left[t_{0}-r, t_{0}\right)
\end{array}
$$


and Henry-Gronwall type retarded integral inequality with singular kernel

$$
\begin{gathered}
u(t) \leq a(t)+\int_{t_{0}}^{t}(t-s)^{\beta-1}[b(s) u(s)+c(s) u(s-r)] d s, \\
t \in\left[t_{0}, T\right), \\
u(t) \leq \phi(t), \quad t \in\left[t_{0}-r, t_{0}\right) .
\end{gathered}
$$

In this paper, motivated by $[6,20]$, we discuss the nonlinear integral inequality of Henry-Gronwall type with delay

$$
\begin{gathered}
u(t) \leq a(t)+\int_{t_{0}}^{t}[b(s) w(u(s))+c(s) w(u(s-r))] d s, \\
t \in\left[t_{0}, T\right), \\
u(t) \leq \phi(t), \quad t \in\left[t_{0}-r, t_{0}\right)
\end{gathered}
$$

and Henry-Gronwall type nonlinear retarded integral inequality with singular kernel

$$
\begin{gathered}
u(t) \\
\leq a(t)+\int_{t_{0}}^{t}(t-s)^{\beta-1}[b(s) w(u(s))+c(s) w(u(s-r))] d s \\
u(t) \leq \phi(t), \quad t \in\left[t_{0}, T\right),
\end{gathered}
$$

\section{Main Results}

Throughout this paper, $\mathbf{R}$ denotes the set of real numbers, $\mathbf{R}_{+}=[0,+\infty)$. For convenience, before giving our main results, we cite some useful lemmas and definitions in the discussion of our proof as follows.

Definition 1 (see [6]). Let $q>0$ be a real number and $0<T \leq$ $\infty$. We say that a function $w: \mathbf{R}_{+} \rightarrow \mathbf{R}_{+}$satisfies a condition (q), if

$$
e^{-q t}[w(u)]^{q} \leq R(t) w\left(e^{-q t} u^{q}\right), \quad \forall u \in \mathbf{R}_{+}, t \in[0, T),
$$

where $R(t)$ is a continuous, nonnegative function.

Lemma 2 (discrete Jensen inequality [25]). Let $A_{1}, A_{2}, \ldots$, $A_{n}$ be nonnegative real numbers, $l>1$ is real numbers, and $n$ is a natural number. Then

$$
\left(A_{1}+A_{2}+\cdots+A_{n}\right)^{l} \leq n^{l-1}\left(A_{1}^{l}+A_{2}^{l}+\cdots+A_{n}^{l}\right) .
$$

Lemma 3 (see [6]). (1) Let $\beta>1 / 2$; then

$$
\int_{t_{0}}^{t}(t-s)^{2 \beta-2} e^{2 s} d s \leq \frac{2 e^{2 t}}{4^{\beta}} \Gamma(2 \beta-1), \quad t_{0}, t \in \mathbf{R}_{+},
$$

where $\Gamma(\beta):=\int_{0}^{\infty} \tau^{\beta-1} e^{-\tau} d \tau$ is the gamma function.
(2) Let $\beta \in(0,1 / 2], p=1+\beta$; then

$$
\begin{array}{r}
\int_{t_{0}}^{t}(t-s)^{p(\beta-1)} e^{p s} d s \leq \frac{e^{p t}}{p^{1+p(\beta-1)}} \Gamma(1+p(\beta-1)), \\
t_{0}, t \in \mathbf{R}_{+} .
\end{array}
$$

Proof. (1) Using a change of variables $\tau=t-s$ and $\xi=2 \tau$ successively, we have the estimate

$$
\begin{aligned}
\int_{t_{0}}^{t}(t-s)^{2 \beta-2} e^{2 s} d s & =\int_{0}^{t-t_{0}} \tau^{2 \beta-2} e^{2 t-2 \tau} d \tau \\
& =e^{2 t} \int_{0}^{t-t_{0}} \tau^{2 \beta-2} e^{-2 \tau} d \tau \\
& =\frac{2 e^{2 t}}{4^{\beta}} \int_{0}^{2 t-2 t_{0}} \xi^{2 \beta-2} e^{-\xi} d \xi \\
& \leq \frac{2 e^{2 t}}{4^{\beta}} \Gamma(2 \beta-1) .
\end{aligned}
$$

Since $\beta>1 / 2,2 \beta-1>0$ and $\Gamma(2 \beta-1) \in \mathbf{R}_{+}$.

(2) Using a change of variables $\tau=t-s$ and $\xi=p \tau$ successively, we have the estimate

$$
\begin{aligned}
\int_{t_{0}}^{t}(t-s)^{p(\beta-1)} e^{p s} d s & =\int_{0}^{t-t_{0}} \tau^{p(\beta-1)} e^{p t-p \tau} d \tau \\
& =e^{p t} \int_{0}^{t-t_{0}} \tau^{p(\beta-1)} e^{-p \tau} d \tau \\
& =\frac{e^{p t}}{p^{1+p(\beta-1)}} \int_{0}^{p t-p t_{0}} \xi^{p(\beta-1)} e^{-\xi} d \xi \\
& \leq \frac{e^{p t}}{p^{1+p(\beta-1)}} \Gamma(1+p(\beta-1)) .
\end{aligned}
$$

Since $0<\beta \leq 1 / 2, p<1 /(1-\beta), 1+p(\beta-1)>0$, and $\Gamma(1+p(\beta-1)) \in \mathbf{R}_{+}$.

Theorem 4. Suppose that $a, b, c$ are nonnegative continuous functions on $\left[t_{0}, T\right), \phi$ is a nonnegative continuous function on $\left[t_{0}-r, t_{0}\right), a\left(t_{0}\right)=\phi\left(t_{0}\right)$, and $t_{0} \geq 0, r>0, T>0$ are constants. Suppose that the function $w$ satisfies the following conditions:

(1) (q) condition, that is, $w$ satisfies inequality (7);

(2) subadditivity, that is, for all $t, s \in \mathbf{R}_{+}, w(t+s) \leq w(t)+$ $w(s)$. 
If $u$ satisfies (5), then

$$
\begin{array}{r}
u(t) \leq a(t)+W^{-1}\left[W\left(\int_{t_{0}}^{t} G(s) d s\right)+\int_{t_{0}}^{t} b(s) d s\right], \\
t \in\left[t_{0}, t_{0}+r\right), \\
u(t) \leq a(t)+W^{-1} \\
\times\left\{W \left[W^{-1}\left(W\left(\int_{t_{0}}^{t_{0}+r} G(s) d s\right)+\int_{t_{0}}^{t_{0}+r} b(s) d s\right)\right.\right. \\
\left.\left.+\int_{t_{0}+r}^{t} H(s) d s\right]+\int_{t_{0}+r}^{t}(b(s)+c(s)) d s\right\}, \\
t \in\left[t_{0}+r, T\right),
\end{array}
$$

where

$$
\begin{gathered}
W(t):=\int_{c}^{t} \frac{d s}{w(s)}, \quad t \in(0, \infty), c>0, W(\infty)=\infty, \quad(14) \\
G(t):=b(t) w(a(t))+c(t) w(\phi(t-r)), \quad t \in\left[t_{0}, t_{0}+r\right),
\end{gathered}
$$

$H(t):=b(t) w(a(t))+c(t) w(a(t-r)), \quad t \in\left[t_{0}+r, T\right)$.

Proof. Define a function $z(t)$ by the right side of (5), that is,

$$
\begin{array}{r}
z(t)=\int_{t_{0}}^{t}[b(s) w(u(s))+c(s) w(u(s-r))] d s, \\
t \in\left[t_{0}, T\right) .
\end{array}
$$

Then $z\left(t_{0}\right)=0, u(t) \leq a(t)+z(t)$, and $z(t)$ is a nonnegative, nondecreasing, and continuous function with $z^{\prime}(t)=$ $b(t) w(u(t))+c(t) w(u(t-r)), t \in\left[t_{0}, T\right)$.

For $t \in\left[t_{0}, t_{0}+r\right)$, by the subadditivity satisfied by $w$, we conclude

$$
\begin{aligned}
z^{\prime}(t) & \leq b(t) w(a(t)+z(t))+c(t) w(\phi(t-r)) \\
& \leq b(t) w(a(t))+c(t) w(\phi(t-r))+b(t) w(z(t)) .
\end{aligned}
$$

Letting $s=t$ in (18) and integrating both sides of inequality (18) from $t_{0}$ to $t$, we obtain

$$
\begin{aligned}
z(t) \leq & \int_{t_{0}}^{t}[b(s) w(a(s))+c(s) w(\phi(s-r))] d s \\
& +\int_{t_{0}}^{t} b(s) w(z(s)) d s \\
\leq & \int_{t_{0}}^{\xi}[b(s) w(a(s))+c(s) w(\phi(s-r))] d s \\
& +\int_{t_{0}}^{t} b(s) w(z(s)) d s, \quad t_{0} \leq t \leq \xi,
\end{aligned}
$$

where $t_{0} \leq \xi \leq t_{0}+r$ is chosen arbitrarily.
Define a function $z_{1}(t)$ by the right side of (19), that is,

$$
\begin{aligned}
z_{1}(t)= & \int_{t_{0}}^{\xi}[b(s) w(a(s))+c(s) w(\phi(s-r))] d s \\
& +\int_{t_{0}}^{t} b(s) w(z(s)) d s, \quad t_{0} \leq t \leq \xi .
\end{aligned}
$$

Then, the function $z_{1}$ is a nonnegative, nondecreasing, and continuous function with

$$
\begin{array}{r}
z_{1}\left(t_{0}\right)=\int_{t_{0}}^{\xi}[b(s) w(a(s))+c(s) w(\phi(s-r))] d s, \\
z(t) \leq z_{1}(t), \quad t_{0} \leq t \leq \xi .
\end{array}
$$

Differentiating $z_{1}$, we have

$$
z_{1}^{\prime}(t) \leq b(t) w\left(z_{1}(t)\right), \quad t_{0} \leq t \leq \xi .
$$

From (22), we obtain

$$
\frac{d z_{1}(t)}{w\left(z_{1}(t)\right)} \leq b(t) d t, \quad t_{0} \leq t \leq \xi .
$$

Using (21), from (23) we obtain

$$
\begin{aligned}
W\left(z_{1}(t)\right) & \leq W\left(z_{1}\left(t_{0}\right)\right)+\int_{t_{0}}^{t} b(s) d s \\
& \leq W\left(\int_{t_{0}}^{\xi} G(s) d s\right)+\int_{t_{0}}^{t} b(s) d s, \quad t_{0} \leq t \leq \xi,
\end{aligned}
$$

where $W, G$ are defined by (14) and (15), respectively. From (24), we observe

$$
z_{1}(t) \leq W^{-1}\left[W\left(\int_{t_{0}}^{\xi} G(s) d s\right)+\int_{t_{0}}^{t} b(s) d s\right], \quad t_{0} \leq t \leq \xi .
$$

Let $t=\xi$ in (25); we have

$$
z_{1}(\xi) \leq W^{-1}\left[W\left(\int_{t_{0}}^{\xi} G(s) d s\right)+\int_{t_{0}}^{\xi} b(s) d s\right] .
$$

Since $\xi$ is chosen arbitrarily, from (26), we have the estimation

$$
\begin{array}{r}
z(t) \leq z_{1}(t) \\
\leq W^{-1}\left[W\left(\int_{t_{0}}^{t} G(s) d s\right)+\int_{t_{0}}^{t} b(s) d s\right], \\
t \in\left[t_{0}, t_{0}+r\right) .
\end{array}
$$

For $t \in\left[t_{0}+r, T\right)$, using the subadditivity of $w$ and monotony of $w, z$, from (17) we have

$$
\begin{aligned}
z^{\prime}(t) \leq & b(t) w(a(s)+z(t))+c(t) w(a(t-r)+z(t-r)) \\
\leq & b(t) w(a(t))+c(t) w(a(t-r))+b(t) w(z(t)) \\
& +c(t) w(z(t-r)) \\
\leq & b(t) w(a(t))+c(t) w(a(t-r))+(b(t)+c(t)) \\
& \times w(z(t)) .
\end{aligned}
$$


Letting $s=t$ in (28) and integrating both sides of inequality (28) from $t_{0}$ to $t$ and using (27) we obtain

$$
\begin{aligned}
z(t) \leq & z\left(t_{0}+r\right)+\int_{t_{0}+r}^{t} H(s) d s \\
& +\int_{t_{0}+r}^{t}(b(s)+c(s)) w(z(s)) d s \\
\leq & W^{-1}\left[W\left(\int_{t_{0}}^{t_{0}+r} G(s) d s\right)+\int_{t_{0}}^{t_{0}+r} b(s) d s\right] \\
& +\int_{t_{0}+r}^{\xi} H(s) d s+\int_{t_{0}+r}^{t}(b(s)+c(s)) w(z(s)) d s, \\
&
\end{aligned}
$$

where $t_{0}+r \leq \xi \leq T, \xi$ is seen as a constant, and $H(s)$ is defined by (16).

Define a function $z_{2}$ by the right side of (29), that is,

$$
\begin{array}{r}
z_{2}(t)=W^{-1}\left[W\left(\int_{t_{0}}^{t_{0}+r} G(s) d s\right)+\int_{t_{0}}^{t_{0}+r} b(s) d s\right] \\
+\int_{t_{0}+r}^{\xi} H(s) d s+\int_{t_{0}+r}^{t}(b(s)+c(s)) w(z(s)) d s \\
t \in\left[t_{0}+r, \xi\right] .
\end{array}
$$

Obviously, $z_{2}$ is a nonnegative, nondecreasing, and continuous function with

$$
\begin{aligned}
z_{2}\left(t_{0}+r\right)= & W^{-1}\left[W\left(\int_{t_{0}}^{t_{0}+r} G(s) d s\right)+\int_{t_{0}}^{t_{0}+r} b(s) d s\right] \\
& +\int_{t_{0}+r}^{\xi}[b(s) w(a(s))+c(s) w(a(s-r))] d s \\
& z(t) \leq z_{2}(t), \quad t_{0}+r \leq t \leq \xi
\end{aligned}
$$

Differentiating $z_{2}$, we have

$$
z_{2}^{\prime}(t) \leq(b(t)+c(t)) w\left(z_{2}(t)\right), \quad t_{0}+r \leq t \leq \xi .
$$

From (33), we have

$$
\frac{d z_{2}(t)}{w\left(z_{2}(t)\right)} \leq(b(t)+c(t)) d t, \quad t_{0}+r \leq t \leq \xi .
$$

Using (31), from (34), we have

$$
\begin{aligned}
& W\left(z_{2}(t)\right) \leq W\left(z_{2}\left(t_{0}+r\right)\right)+\int_{t_{0}+r}^{t}(b(s)+c(s)) d s \\
& \leq W\left\{W^{-1}\left[W\left(\int_{t_{0}}^{t_{0}+r} G(s) d s\right)+\int_{t_{0}}^{t_{0}+r} b(s) d s\right]\right. \\
&\left.+\int_{t_{0}+r}^{\xi} H(s) d s\right\}+\int_{t_{0}+r}^{t}(b(s)+c(s)) d s \\
& t \in\left[t_{0}+r, \xi\right] .
\end{aligned}
$$

It follows that

$$
\begin{aligned}
& z_{2}(t) \leq W^{-1} \\
& \times\left\{W \left\{W^{-1}\left[W\left(\int_{t_{0}}^{t_{0}+r} G(s) d s\right)+\int_{t_{0}}^{t_{0}+r} b(s) d s\right]\right.\right. \\
&\left.\left.\quad+\int_{t_{0}+r}^{\xi} H(s) d s\right\}+\int_{t_{0}+r}^{t}(b(s)+c(s)) d s\right\}, \\
& t \in\left[t_{0}+r, \xi\right] .
\end{aligned}
$$

In (36), let $t=\xi$, and then we have

$z_{2}(\xi)$

$$
\begin{array}{r}
\leq W^{-1}\left\{W \left[W^{-1}\left(W\left(\int_{t_{0}}^{t_{0}+r} G(s) d s\right)+\int_{t_{0}}^{t_{0}+r} b(s) d s\right)\right.\right. \\
\left.\left.+\int_{t_{0}+r}^{\xi} H(s) d s\right]+\int_{t_{0}+r}^{\xi}(b(s)+c(s)) d s\right\} .
\end{array}
$$

Since $\xi$ is chosen arbitrarily, from (32) and (37), we obtain the estimation

$$
\begin{array}{r}
z(t) \leq W^{-1}\left\{W \left[W^{-1}\left(W\left(\int_{t_{0}}^{t_{0}+r} G(s) d s\right)+\int_{t_{0}}^{t_{0}+r} b(s) d s\right)\right.\right. \\
\left.\left.+\int_{t_{0}+r}^{t} H(s) d s\right]+\int_{t_{0}+r}^{t}(b(s)+c(s)) d s\right\}, \\
t \in\left[t_{0}+r, T\right) .
\end{array}
$$

Noting that $u(t) \leq a(t)+z(t)$, from (27) and (38), we obtain our required estimations (13).

Remark 5. When $w(u(t))=u(t)$. The estimations (13) in Theorem 4 are reduced to the corresponding estimations in [20].

Theorem 6. Suppose that $a, b, c, u, w, \phi, r$ satisfy the corresponding conditions in Theorem $4 ; \beta$ is a constant. If u satisfies (6), then the following assertions hold.

(1) Suppose $\beta>1 / 2$. Then

$$
\begin{aligned}
z(t) \leq & A(t)+W^{-1}\left[W\left(\int_{t_{0}}^{t} I(s) d s\right)+\int_{t_{0}}^{t} B(s) d s\right], \\
t \in\left[t_{0}, t_{0}+r\right), & \\
z(t) \leq & A(t)+W^{-1} \\
\times & \left\{W \left[W^{-1}\left(W\left(\int_{t_{0}}^{t_{0}+r} I(s) d s\right)+\int_{t_{0}}^{t_{0}+r} B(s) d s\right)\right.\right. \\
& \left.\left.+\int_{t_{0}+r}^{t} J(s) d s\right]+\int_{t_{0}+r}^{t}(B(s)+C(s)) d s\right\}, \\
& t \in\left[t_{0}+r, T\right),
\end{aligned}
$$


where $W$ is defined by (14) in Theorem 4,

$I(s):=B(t) w(A(t))+C(t) w(\Phi(t-r)), \quad t \in\left[t_{0}, t_{0}+r\right)$,

$$
J(t):=B(t) w(A(t))+C(t) w(A(t-r)), \quad t \in\left[t_{0}+r, T\right),
$$

$$
\begin{aligned}
A(t) & :=\max \left\{3, e^{2 r}\right\}\left[e^{t_{0}} a(t)\right]^{2}, \quad t \in\left[t_{0}, T\right), \\
B(t) & :=\frac{6}{4^{\beta}} \Gamma(2 \beta-1) b^{2}(t) R(t), \quad t \in\left[t_{0}, T\right), \\
C(t) & :=\frac{6}{4^{\beta}} \Gamma(2 \beta-1) e^{-2 r} c^{2}(t) R(t-r), \quad t \in\left[t_{0}, T\right),
\end{aligned}
$$$$
\Phi(t):=\max \left\{3, e^{2 r}\right\}\left[e^{-t_{0}} \phi(t)\right]^{2}, \quad t \in\left[t_{0}, T\right),
$$

and $R(t)$ is defined in (7) in Definition 1.

(2) Suppose that $\beta \in(0,1 / 2], p=1+\beta$. Then

$$
\begin{gathered}
z(t) \leq D(t)+W^{-1}\left[W\left(\int_{t_{0}}^{t} K(s) d s\right)+\int_{t_{0}}^{t} E(s) d s\right], \\
t \in\left[t_{0}, t_{0}+r\right), \\
z(t) \leq D(t)+W^{-1} \\
\left\{W \left[W^{-1}\left(W\left(\int_{t_{0}}^{t_{0}+r} K(s) d s\right)+\int_{t_{0}}^{t_{0}+r} E(s) d s\right)\right.\right. \\
\left.\left.+\int_{t_{0}+r}^{t} L(s) d s\right]+\int_{t_{0}+r}^{t}(E(s)+F(s)) d s\right\}, \\
t \in\left[t_{0}+r, T\right),
\end{gathered}
$$

where

$$
\begin{gathered}
K(s):=E(t) w(D(t))+F(t) w(\Psi(t-r)), \\
t \in\left[t_{0}, t_{0}+r\right), \\
L(t):=E(t) w(D(t))+F(t) w(D(t-r)), \\
t \in\left[t_{0}+r, T\right), \\
E(t):=3\left[\frac{1}{\left.p^{1+p(\beta-1)} \Gamma(1+p(\beta-1))\right]^{q / p} b^{q}(t) R(t),}\right. \\
F(t):=\rho\left[e^{t_{0}} a(t)\right]^{q}, \quad t \in\left[t_{0}, T\right), \\
\left.\quad t=3\left[\frac{1}{p^{1+p(\beta-1)}} \Gamma(1+p(\beta-1))\right]^{q / p}, T\right), \\
\times e^{-q r} c^{q}(t) R(t-r), \quad t \in\left[t_{0}, T\right), \\
\Psi(t):=\rho\left[e^{-t_{0}} \phi(t)\right]^{q}, \quad t \in\left[t_{0}, T\right),
\end{gathered}
$$

and $q=1+1 / \beta$.
Proof. First we will prove assertion (1). Suppose that $\beta>1 / 2$. Using Cauchy-Schwarz inequality, we obtain from (6) that

$$
\begin{aligned}
u(t) \leq & a(t)+\int_{t_{0}}^{t}(t-s)^{\beta-1} e^{s} b(s) e^{-s} w(u(s)) d s \\
& +\int_{t_{0}}^{t}(t-s)^{\beta-1} e^{s-r} c(s) e^{-s+r} w(u(s-r)) d s \\
\leq & a(t)+\left[\int_{t_{0}}^{t}(t-s)^{2 \beta-2} e^{2 s} d s\right]^{1 / 2} \\
& \times\left[\int_{t_{0}}^{t} b^{2}(s) e^{-2 s} w^{2}(u(s)) d s\right]^{1 / 2} \\
& +\left[\int_{t_{0}}^{t}(t-s)^{2 \beta-2} e^{2 s} d s\right]^{1 / 2} \\
& \times\left[\int_{t_{0}}^{t} e^{-2 r} c^{2}(s) e^{-2 s+2 r} w^{2}(u(s-r)) d s\right]^{1 / 2},
\end{aligned}
$$

Since $w$ satisfies $(q)$ condition, using (7) in Definition 1 and (9) in Lemma 3, from (53) we derive that

$$
\begin{aligned}
u(t) \leq & a(t)+\left[\frac{2 e^{2 t}}{4^{\beta}} \Gamma(2 \beta-1)\right]^{1 / 2} \\
& \times\left[\int_{t_{0}}^{t} b^{2}(s) R(s) w\left(u^{2}(s) e^{-2 s}\right) d s\right]^{1 / 2} \\
& +\left[\frac{2 e^{2 t}}{4^{\beta}} \Gamma(2 \beta-1)\right]^{1 / 2} \\
& \times\left[\int_{t_{0}}^{t} e^{-2 r} c^{2}(s) R(s-r) w\left(u^{2}(s-r) e^{-2 s+2 r}\right) d s\right]^{1 / 2}
\end{aligned}
$$

for all $t \in\left[t_{0}, T\right)$. Using discrete Jensen inequality (8) with $n=3, l=2$, from (54) we obtain

$$
\begin{aligned}
& u^{2}(t) \leq 3 a^{2}(t)+3 \frac{2 e^{2 t}}{4^{\beta}} \Gamma(2 \beta-1) \\
& \times\left[\int_{t_{0}}^{t} b^{2}(s) R(s) w\left(u^{2}(s) e^{-2 s}\right) d s\right. \\
&\left.+\int_{t_{0}}^{t} e^{-2 r} c^{2}(s) R(s-r) w\left(u^{2}(s-r) e^{-2 s+2 r}\right) d s\right], \\
& t \in\left[t_{0}, T\right) .
\end{aligned}
$$


Let $v(t)=\left[e^{-t} u(t)\right]^{2}$ and $\lambda=\max \left\{3, e^{2 r}\right\}$. From (55) we have

$$
\begin{aligned}
v(t) \leq \lambda\left[e^{t_{0}} a(t)\right]^{2}+\frac{6}{4 \beta} \Gamma(2 \beta-1) & \\
\times & {\left[\int_{t_{0}}^{t} b^{2}(s) R(s) w(v(s)) d s\right.} \\
& \left.+\int_{t_{0}}^{t} e^{-2 r} c^{2}(s) R(s-r) w(v(s-r)) d s\right], \\
& t \in\left[t_{0}, T\right) .
\end{aligned}
$$

We observe that

$$
\begin{aligned}
v(t) & =\left[e^{-t} \phi(t)\right]^{2} \leq e^{2 r}\left[e^{-t_{0}} \phi(t)\right]^{2} \\
& \leq \lambda\left[e^{-t_{0}} \phi(t)\right]^{2}=\Phi(t), \quad t \in\left[t_{0}-r, t_{0}\right),
\end{aligned}
$$

$\Phi(t)$ is defined by (45). By the definitions of $A(t), B(t)$, and $C(t)$ in (42), (43), and (44), from (56) we see

$$
\begin{aligned}
v(t) \leq & A(t)+\int_{t_{0}}^{t} B(s) w(v(s)) d s \\
& +\int_{t_{0}}^{t} C(s) w(v(s-r)) d s, \quad t \in\left[t_{0}, T\right) ; \\
& v(t) \leq \Phi(t), \quad t \in\left[t_{0}-r, t_{0}\right) .
\end{aligned}
$$

We observe that (58) have the same form as (5) and $A(t), B(t), C(t)$ satisfy the corresponding conditions in Theorem 4. Applying Theorem 4 to (58), we obtain our required estimations (39).

(2) Now let us prove assertion (2). Suppose $\beta \epsilon$ $(0,1 / 2], p=1+\beta$. Let $q=1+1 / \beta$; then $1 / p+1 / q=1$. Using Hölder inequality, from (6) we obtain

$$
\begin{aligned}
u(t) \leq & a(t)+\int_{t_{0}}^{t}(t-s)^{\beta-1} e^{s} b(s) e^{-s} w(u(s)) d s \\
& +\int_{t_{0}}^{t}(t-s)^{\beta-1} e^{s-r} c(s) e^{-s+r} w(u(s-r)) d s \\
\leq & a(t)+\left[\int_{t_{0}}^{t}(t-s)^{p \beta-p} e^{p s} d s\right]^{1 / p} \\
& \times\left[\int_{t_{0}}^{t} b^{q}(s) e^{-q s} w^{q}(u(s)) d s\right]^{1 / q} \\
& +\left[\int_{t_{0}}^{t}(t-s)^{p \beta-p} e^{p s} d s\right]^{1 / p} \\
& \times\left[\int_{t_{0}}^{t} e^{-q r} c^{q}(s) e^{-q s+q r} w^{q}(u(s-r)) d s\right]^{1 / q}, \\
& \quad t \in\left[t_{0}, T\right) .
\end{aligned}
$$

Since $w$ satisfies (q) condition, using (7) and (10), from (59) we derive

$$
\begin{aligned}
u(t) \leq & a(t)+\left[\frac{e^{p t}}{p^{1+p(\beta-1)}} \Gamma(1+p(\beta-1))\right]^{1 / p} \\
& \times\left[\int_{t_{0}}^{t} b^{q}(s) R(s) w\left(u^{q}(s) e^{-q s}\right) d s\right]^{1 / q} \\
& +\left[\frac{e^{p t}}{p^{1+p(\beta-1)}} \Gamma(1+p(\beta-1))\right]^{1 / p} \\
& \times\left[\int_{t_{0}}^{t} e^{-q r} c^{q}(s) R(s-r) w\left(u^{q}(s-r) e^{-q s+q r}\right) d s\right]^{1 / q},
\end{aligned}
$$

for all $t \in\left[t_{0}, T\right)$. Using Jensen inequality (8), from (60) we have

$$
\begin{array}{rl}
u^{q}(t) \leq & 3 a^{q}(t)+3\left[\frac{e^{p t}}{p^{1+p(\beta-1)}} \Gamma(1+p(\beta-1))\right]^{q / p} \\
\times & {\left[\int_{t_{0}}^{t} b^{q}(s) R(s) w\left(u^{q}(s) e^{-q s}\right) d s\right.} \\
& \left.+\int_{t_{0}}^{t} e^{-q r} c^{q}(s) R(s-r) w\left(u^{q}(s-r) e^{-q s+q r}\right) d s\right], \\
t & t \in\left[t_{0}, T\right) .
\end{array}
$$

Let $v(t)=\left[e^{-t} u(t)\right]^{q}$ and $\rho=\max \left\{3, e^{q r}\right\}$. Then, we obtain from (61) that

$$
\begin{aligned}
v(t) \leq \rho\left[e^{t_{0}} a(t)\right]^{q}+3\left[\frac{1}{p^{1+p(\beta-1)}} \Gamma(1+p(\beta-1))\right]^{q / p} \\
\times\left[\int_{t_{0}}^{t} b^{q}(s) R(s) w\left(u^{q}(s) e^{-q s}\right) d s\right. \\
\left.+\int_{t_{0}}^{t} e^{-q r} c^{q}(s) R(s-r) w\left(u^{q}(s-r) e^{-q s+q r}\right) d s\right], \\
t \in\left[t_{0}, T\right) .
\end{aligned}
$$

We observe that

$$
\begin{aligned}
v(t) & =\left[e^{-t} \phi(t)\right]^{q} \leq e^{q r}\left[e^{-t_{0}} \phi(t)\right]^{q} \\
& \leq \rho\left[e^{-t_{0}} \phi(t)\right]^{q}=\Psi(t), \quad t \in\left[t_{0}-r, t_{0}\right),
\end{aligned}
$$

where $\Psi(t)$ is defined by (52). Using definitions of $D(t), E(t)$ and $F(t)$ in (49), (50), and (51), from (62) we have

$$
\begin{aligned}
v(t) \leq & D(t)+\int_{t_{0}}^{t} E(s) w(v(s)) d s \\
& +\int_{t_{0}}^{t} F(s) w(v(s-r)) d s, \quad t \in\left[t_{0}, T\right), \\
& v(t) \leq \Psi(t), \quad t \in\left[t_{0}-r, t_{0}\right) .
\end{aligned}
$$


We observe that (64) have the same form as (5) and $D(t)$, $E(t), F(t)$ satisfy the corresponding conditions in Theorem 4. Applying Theorem 4 to (64), we obtain our required estimations (46).

\section{Application to Fractional Differential Equations (FDEs) with Delay}

In this section, we apply our result to the following fractional differential equations (FDEs) with delay (see [20]):

$$
\begin{gathered}
D^{\beta} x(t)=f(t, x(t), x(t-r)), \quad t \in\left[t_{0}, T\right), \\
x(t)=\phi(t), \quad t \in\left[t_{0}-r, t_{0}\right),
\end{gathered}
$$

where $D^{\beta}$ represents the Caputo fractional derivative of order $\beta(\beta>0), f \in C\left(\left[t_{0}, T\right) \times \mathbf{R} \times \mathbf{R}, \mathbf{R}\right)$, and $\phi$ is as in Theorem 6 .

Theorem 7. Suppose that

$$
|f(t, x, y)| \leq b(t) w(|x|)+c(t) w(|y|),
$$

where $b(t), c(t), w$ are as in Theorem 6. Let $M=\max _{t \in\left[t_{0}-r, t_{0}\right)}$ $|\phi(t)|$. If $x(t)$ is any solution of IVP (65), then the following estimates hold.

(1) Suppose $1 / 2<\beta \leq 1$. Then

$$
\begin{gathered}
z(t) \leq A(t)+W^{-1}\left[W\left(\int_{t_{0}}^{t} \widetilde{I}(s) d s\right)+\int_{t_{0}}^{t} \widetilde{B}(s) d s\right], \\
t \in\left[t_{0}, t_{0}+r\right), \\
z(t) \leq A(t)+W^{-1}\left\{W \left[W ^ { - 1 } \left(W\left(\int_{t_{0}}^{t_{0}+r} \widetilde{I}(s) d s\right)\right.\right.\right. \\
\left.+\int_{t_{0}}^{t_{0}+r} \widetilde{B}(s) d s\right) \\
\left.+\int_{t_{0}+r}^{t} \widetilde{J}(s) d s\right] \\
\left.+\int_{t_{0}+r}^{t}(\widetilde{B}(s)+\widetilde{C}(s)) d s\right\}, \\
t \in\left[t_{0}+r, T\right),
\end{gathered}
$$

where $W$ is defined by (14) in Theorem 4 ,

$$
\begin{gathered}
\widetilde{I}(s):=\widetilde{B}(t) w(\widetilde{A}(t))+\widetilde{C}(t) w(\Phi(t-r)), \\
t \in\left[t_{0}, t_{0}+r\right), \\
\widetilde{J}(t):=\widetilde{B}(t) w(\widetilde{A}(t))+\widetilde{C}(t) w(\widetilde{A}(t-r)), \\
t \in\left[t_{0}+r, T\right), \\
\widetilde{A}(t):=\max \left\{3, e^{2 r}\right\}\left[e^{t_{0}} M\right]^{2}, \quad t \in\left[t_{0}, T\right), \\
\widetilde{B}(t):=\frac{6 \Gamma(2 \beta-1) b^{2}(t) R(t)}{\left(4^{\beta} \Gamma(\beta)\right)}, \quad t \in\left[t_{0}, T\right), \\
\widetilde{C}(t):=\frac{6 \Gamma(2 \beta-1) e^{-2 r} c^{2}(t) R(t-r)}{\left(4^{\beta} \Gamma(\beta)\right)}, \quad t \in\left[t_{0}, T\right),
\end{gathered}
$$

and $R(t), \Phi$ are defined by (7) and (45), respectively.
(2) Suppose that $\beta \in(0,1 / 2], p=1+\beta$. Then

$$
\begin{gathered}
z(t) \leq D(t)+W^{-1}\left[W\left(\int_{t_{0}}^{t} \widetilde{K}(s) d s\right)+\int_{t_{0}}^{t} \widetilde{E}(s) d s\right], \\
t \in\left[t_{0}, t_{0}+r\right), \\
z(t) \leq D(t) \\
+W^{-1}\left\{W \left[W ^ { - 1 } \left(W\left(\int_{t_{0}}^{t_{0}+r} \widetilde{K}(s) d s\right)\right.\right.\right. \\
\left.\left.+\int_{t_{0}}^{t_{0}+r} \widetilde{E}(s) d s\right)+\int_{t_{0}+r}^{t} \widetilde{L}(s) d s\right] \\
\left.+\int_{t_{0}+r}^{t}(\widetilde{E}(s)+\widetilde{F}(s)) d s\right\}, \\
t \in\left[t_{0}+r, T\right),
\end{gathered}
$$

where

$$
\begin{aligned}
& \widetilde{K}(s):=\widetilde{E}(t) w(\widetilde{D}(t))+\widetilde{F}(t) w(\Psi(t-r)), t \in\left[t_{0}, t_{0}+r\right), \\
& \widetilde{L}(t):= \widetilde{E}(t) w(\widetilde{D}(t))+\widetilde{F}(t) w(\widetilde{D}(t-r)), \\
& t \in\left[t_{0}+r, T\right), \\
& \widetilde{D}(t):=\rho\left[e^{t_{0}} M\right]^{q}, \quad t \in\left[t_{0}, T\right), \\
& \widetilde{E}(t):=3\left[\frac{1}{p^{1+p(\beta-1)}} \Gamma(1+p(\beta-1))\right]^{q / p} \\
& \times\left(\frac{b(t)}{\Gamma(\beta)}\right)^{q} R(t), \quad t \in\left[t_{0}, T\right), \\
& \widetilde{F}(t):=3\left[\frac{1}{p^{1+p(\beta-1)}} \Gamma(1+p(\beta-1))\right]^{q / p} \\
& \times\left(\frac{c(t)}{\Gamma(\beta)}\right)^{q} e^{-q r} R(t-r), \quad t \in\left[t_{0}, T\right),
\end{aligned}
$$

and $q=1+1 / \beta ; \Psi(t)$ is defined by $(52)$.

Proof. The solution $x(t)$ of FDEs (65) can be written as (see [24])

$$
\begin{gathered}
x(t)=\sum_{k=0}^{n-1} \frac{b_{k}}{k !}\left(t-t_{0}\right)^{k}+\frac{1}{\Gamma(\beta)} \\
\times \int_{t_{0}}^{t}(t-s)^{\beta-1} f(s, x(s), x(s-r)) d s, \quad t \in\left[t_{0}, T\right), \\
x(t)=\phi(t), \quad t \in\left[t_{0}-r, t_{0}\right) .
\end{gathered}
$$


When $0<\beta \leq 1$, from (71) we obtain

$$
\begin{aligned}
& |x(t)| \\
& \leq M+\frac{1}{\Gamma(\beta)} \\
& \quad \times \int_{t_{0}}^{t}(t-s)^{\beta-1}[b(s) w(|x(s)|)+c(s) w(|x(s-r)|)] d s, \\
& \quad|x(t)| \leq M, \quad t \in\left[t_{0}, T\right),
\end{aligned}
$$

Applying Theorem 6 to (72), we obtain our required estimations (67) and (69).

Remark 8. When $\beta>1$. Let $\widetilde{D}(t)=M+\sum_{k=0}^{n-1}\left(b_{k} / k !\right)\left(t-t_{0}\right)^{k}$; we can obtain the estimations similar to (67) in Theorem 7.

\section{Conflict of Interests}

The authors declare that they have no competing interests.

\section{Acknowledgments}

This research was supported by the National Natural Science Foundation of China (no. 11161018), the Guangxi Natural Science Foundation (no. 2012GXNSFAA053009), the Scientific Research Foundation of the Education Department of Guangxi Province (no. LX2014330), and the Foundation of Scientific Research Project of Fujian Province Education Department of China (no. JK2012049). The authors would like to thank the anonymous reviewers for their valuable comments and suggestions to improve the quality of the paper.

\section{References}

[1] T. H. Gronwall, "Note on the derivatives with respect to a parameter of the solutions of a system of differential equations," Annals of Mathematics. Second Series, vol. 20, no. 4, pp. 292-296, 1919.

[2] R. Bellman, "The stability of solutions of linear differential equations," Duke Mathematical Journal, vol. 10, pp. 643-647, 1943.

[3] I. A. Bihari, "A generalization of a lemma of Bellman and its application to uniqueness problems of differential equations," Acta Mathematica Academiae Scientiarum Hungaricae, vol. 7, pp. 81-94, 1956.

[4] D. Henry, Geometric Theory of Semilinear Parabolic Equations, Springer, Berlin, Germany, 1981.

[5] H. Sano and N. Kunimatsu, "Modified Gronwall's inequality and its application to stabilization problem for semilinear parabolic systems," Systems \& Control Letters, vol. 22, no. 2, pp. 145-156, 1994.

[6] M. Medved', "A new approach to an analysis of Henry type integral inequalities and their Bihari type versions," Journal of Mathematical Analysis and Applications, vol. 214, no. 2, pp. 349366, 1997.
[7] M. Medved', "Nonlinear singular integral inequalities for functions in two and $n$ independent variables," Journal of Inequalities and Applications, vol. 5, no. 3, pp. 287-308, 2000.

[8] Q. H. Ma and E. H. Yang, "Estimates on solutions of some weakly singular Volterra integral inequalities," Acta Mathematicae Applicatae Sinica, vol. 25, no. 3, pp. 505-515, 2002 (Chinese).

[9] Y. Wu and S. F. Deng, "Generalization of some weakly singular Volterra integral inequalities," Journal of Sichuan University: Natural Science Edition, vol. 41, no. 3, pp. 473-478, 2004 (Chinese).

[10] K. M. Furati and N.-E. Tatar, "Behavior of solutions for a weighted Cauchy-type fractional differential problem," Journal of Fractional Calculus, vol. 28, pp. 23-42, 2005.

[11] R. P. Agarwal, S. Deng, and W. Zhang, "Generalization of a retarded Gronwall-like inequality and its applications," Applied Mathematics and Computation, vol. 165, no. 3, pp. 599-612, 2005.

[12] M. Medved', "On singular versions of Bihari and WendroffPachpatte type integral inequalities and their application," Tatra Mountains Mathematical Publications, vol. 38, pp. 163-174, 2007.

[13] H. Ye, J. Gao, and Y. Ding, "A generalized Gronwall inequality and its application to a fractional differential equation," Journal of Mathematical Analysis and Applications, vol. 328, no. 2, pp. 1075-1081, 2007.

[14] W. Cheung, Q. Ma, and S. Tseng, "Some new nonlinear weakly singular integral inequalities of wendroff type with applications," Journal of Inequalities and Applications, vol. 2008, Article ID 909156, 12 pages, 2008.

[15] Q. Ma and J. Pečarić, "Some new explicit bounds for weakly singular integral inequalities with applications to fractional differential and integral equations," Journal of Mathematical Analysis and Applications, vol. 341, no. 2, pp. 894-905, 2008.

[16] S. Deng and C. Prather, "Generalization of an impulsive nonlinear singular Gronwall-Bihari inequality with delay," Journal of Inequalities in Pure and Applied Mathematics, vol. 9, no. 2, 11 pages, 2008.

[17] Y. Wu, "A new type of weakly singular Volterra integral inequalities," Acta Mathematicae Applicatae Sinica, vol. 31, no. 4, pp. 584-591, 2008.

[18] S. Mazouzi and N. Tatar, "New bounds for solutions of a singular integro-differential inequality," Mathematical Inequalities \& Applications, vol. 13, no. 2, pp. 427-435, 2010.

[19] H. Wang and K. Zheng, "Some nonlinear weakly singular integral inequalities with two variables and applications," Journal of Inequalities and Applications, vol. 2010, Article ID 345701, 12 pages, 2010.

[20] H. Ye and J. Gao, "Henry-Gronwall type retarded integral inequalities and their applications to fractional differential equations with delay," Applied Mathematics and Computation, vol. 218, no. 8, pp. 4152-4160, 2011.

[21] A. Abdeldaim and M. Yakout, "On some new integral inequalities of Gronwall-Bellman-Pachpatte type," Applied Mathematics and Computation, vol. 217, no. 20, pp. 7887-7899, 2011.

[22] Q. H. Ma and E. H. Yang, "Bounds on solutions to some nonlinear Volterra integral inequalities with weakly singular kernels," Annals of Differential Equations, vol. 27, no. 3, pp. 283292, 2011

[23] K. Zheng, "Bounds on some new weakly singular Wendrofftype integral inequalities and applications," Journal of Inequalities and Applications, vol. 2013, article 159, 2013. 
[24] I. Podlubny, Fractional Differential Equations, Academic Press, New York, NY, USA, 1999.

[25] M. Kuczma, An Introduction to the Theory of Functional Equations and Inequalities: Cauchys Equation and Jensens Inequality, University of Katowice, Katowice, Poland, 1985. 


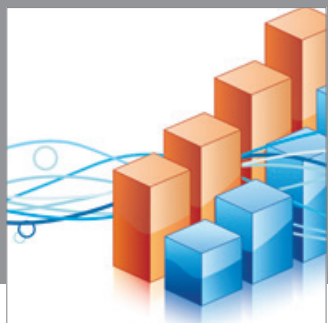

Advances in

Operations Research

mansans

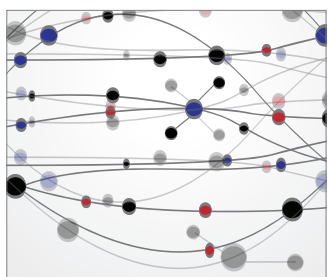

The Scientific World Journal
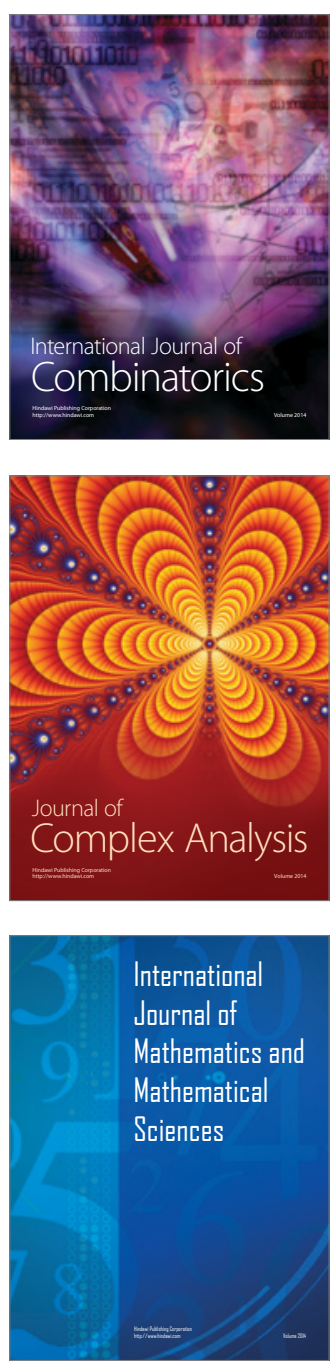
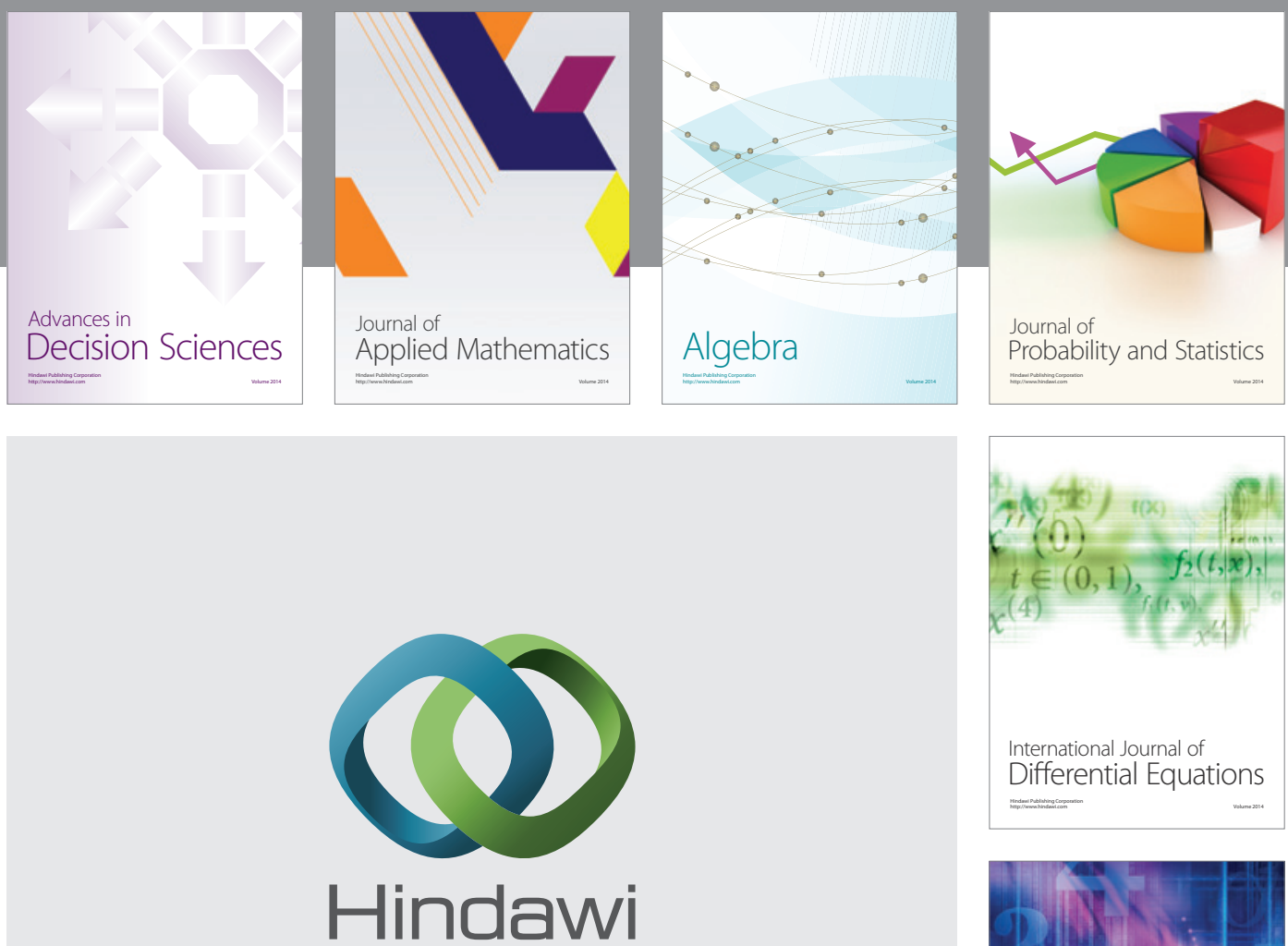

Submit your manuscripts at http://www.hindawi.com
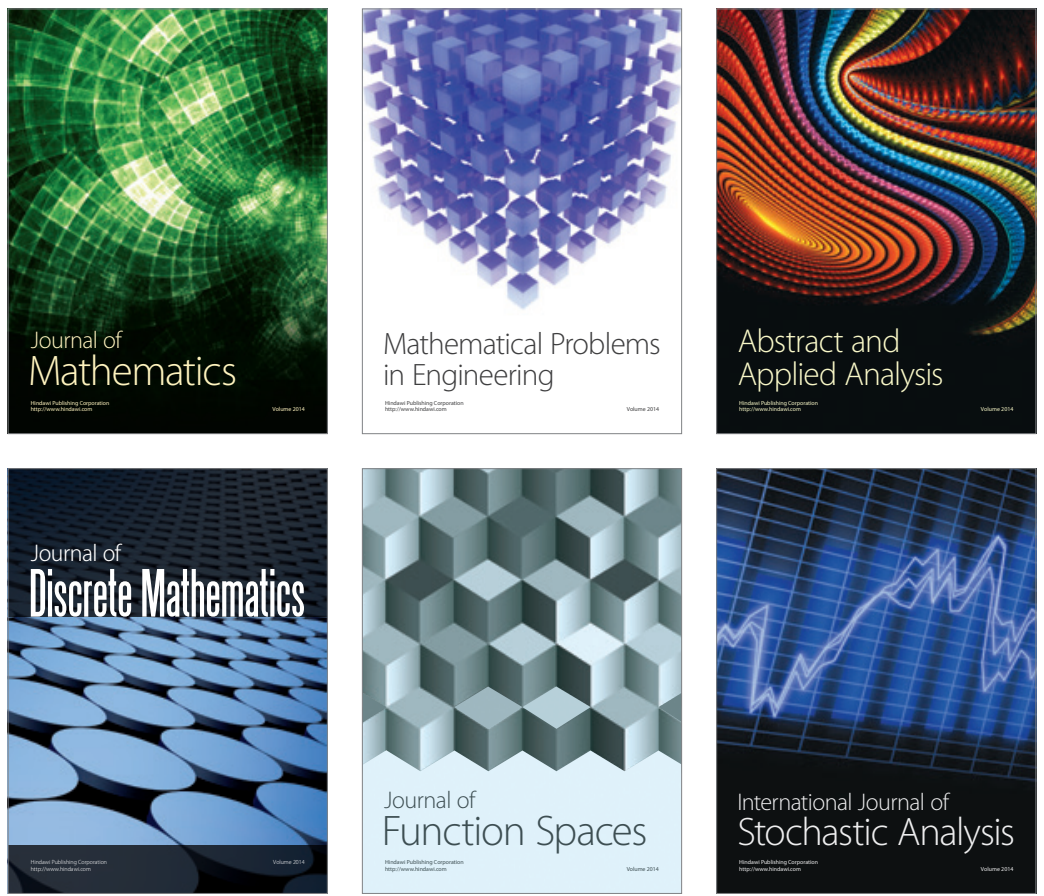

Journal of

Function Spaces

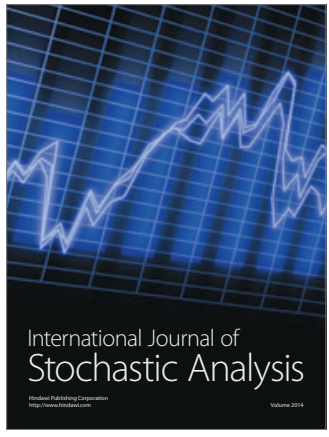

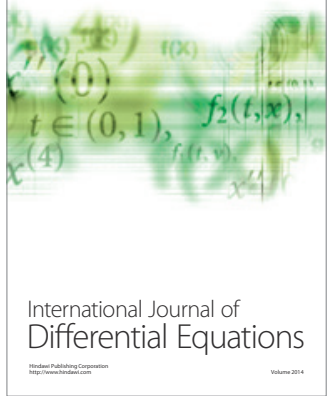
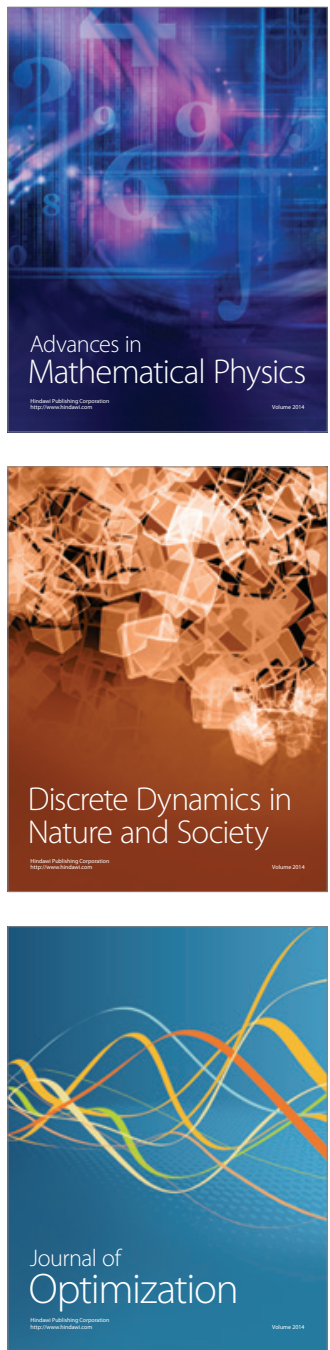